\title{
Experimental observation of Loschmidt time reversal of a Quantum Chaotic System
}

\author{
A. Ullah and M.D. Hoogerland \\ Department of Physics, University of Auckland, Private Bag 92019, Auckland, New Zealand
}

\begin{abstract}
We have performed an experiment to demonstrate the approximate time reversal of a "chaotic" time evolution of atomic deBroglie waves. We use ultra cold atoms from a Bose-Einstein condensate in a quantum $\delta$-kicked rotor experiment, and show that an initial state can be approximately recreated even after a period of "chaotic" evolution (a number of kicks). As this mechanism only works for a very narrow range of momenta, the net effect is a narrowing of the momentum distribution after the kick sequence.
\end{abstract}

PACS numbers: $05.45 . \mathrm{Mt}, 32.80 . \mathrm{Lg}$

How the irreversibility of macroscopic systems is reconciled with the reversibility of microscopic physical laws has been discussed for over a century. The argument was first made by J. Loschmidt [1] in reaction to Boltzmann's statistical theory of gases. These were the ideas which helped to develop the theory of dynamical chaos [2, 3]. Small perturbations may grow exponentially with time in classical dynamics which makes the motion practically irreversible. This phenomenon is known as classical chaos [4, 5]. In quantum dynamics on the other hand, chaos does not exist. Exponential divergence takes place only during the rather short Ehrenfest time [6], and the quantum evolution remains stable and reversible in the presence of small perturbations.

The delta-kicked rotor is a simple system well suited to the study of classical and quantum chaos $7-10]$. As a consequence of the quantum-mechanical nature of the motion, dynamical localization has been observed in this system [11, 12]. It has been central to the study of "quantum chaos", receiving significant attention in recent years [13, 14]. Casati and co-workers predicted that a quantum particle follows the diffusive dynamics of a classical chaotic system only up to a certain time, known as the quantum break time [15, 16]. The classical diffusion ceases due to quantum interference, and the momentum distribution settles into an exponential distribution at this point.

Here we present an experimental realization of the effective time reversal of atomic matter waves as proposed by Martin et. al. [17]. We use a cloud of ultra cold ${ }^{87} \mathrm{Rb}$ atoms, driven by a pulsed optical standing wave, as a quantum $\delta$-kicked rotor system. We show that the effects of a certain number of kicks can be effectively reversed by further kicks by having a different kick sequence. We observe that a significant fraction of the atoms return back to their original zero momentum state. As the time reversal is very sensitive to the original momentum, we observe a narrowing of the zero momentum peak. This phenomenon has been named "Loschmidt cooling" 17], even though the phase space density is not increased. It should be stressed that many schemes exist that narrow the momentum distribution of a sample of atoms 18 $^{-}$ 20], some of which increase the phase space density. The results presented here however, show the first observa- tion of an effective reversal of chaotic dynamics in the quantum regime. The phenomenon observed here can be thought of as a multiple beam interferometry, where the sharpness of the interference fringes due to constructive interference has been demonstrated [21].

The atom optics implementation of the delta-kicked rotor consists of a two-level atom placed in a pulsed standing wave of laser light that is detuned from resonance. The laser field gives rise to a potential that varies sinusoidally with position. The Hamiltonian of the system can be written as

$$
H=\frac{p^{2}}{2 m}-V_{0} \cos \left(k_{L} x\right) f(t),
$$

where $f(t)$ describes the time dependence of the laser pulses. For the delta kicked rotor, $f(t)=\sum_{n=1}^{N} \delta(t-n T)$, where $T$ is the kick period. In this system, it is convenient to use the scaled kick period $\hbar=8 \omega_{R} T$ where $\omega_{R}=$ $\hbar k_{L}^{2} /(2 m)$ is the recoil frequency, with $k_{L}=2 \pi / \lambda, \lambda$ is the wavelength of the laser beam and $m$ the mass of the atom. A "quantum resonance" exists for $k=4 \pi$, where all kicks add coherently, and quadratic energy growth with the number of kicks is observed. At $k=2 \pi$, an "anti-resonance" is observed, where the effect of each kick is effectively negated by the following kick.

The potential $V_{0}$ can be written as $\hbar \phi_{d}$, where $\phi_{d}=$ $\tau_{p} \Omega^{2} / 4 \Delta$ is the kick strength, $\tau_{p}$ is the pulse duration, and $\Omega=d \cdot E /(2 \hbar)$ is the on-resonance Rabi frequency. The parameter $d$ is the atomic dipole moment induced by the laser with electric field $E . \Delta=\omega_{L}-\omega_{0}$ is the detuning from resonance, where $\omega_{L}$ is the laser frequency and $\omega_{0}$ is the resonance frequency.

Following [17] we use a Loschmidt pulse train consisting of $N / 2$ pulses with a scaled period of $k=4 \pi+\epsilon$, a waiting time $6 \pi$ after the last pulse and then $N / 2$ pulses with a period of $4 \pi-\epsilon$, thus a total of $N$ pulses. The parameter $\epsilon$ is proportional to the difference of the pulse period from the first primary resonance.

We simulate the time evolution of the atom optics kicked rotor using the split operator method as described in 22]. We Fourier transform our wave function from position space into momentum space and back again, as the kick potential is a diagonal operator in position space, and the free evolution is a diagonal operator in momen- 


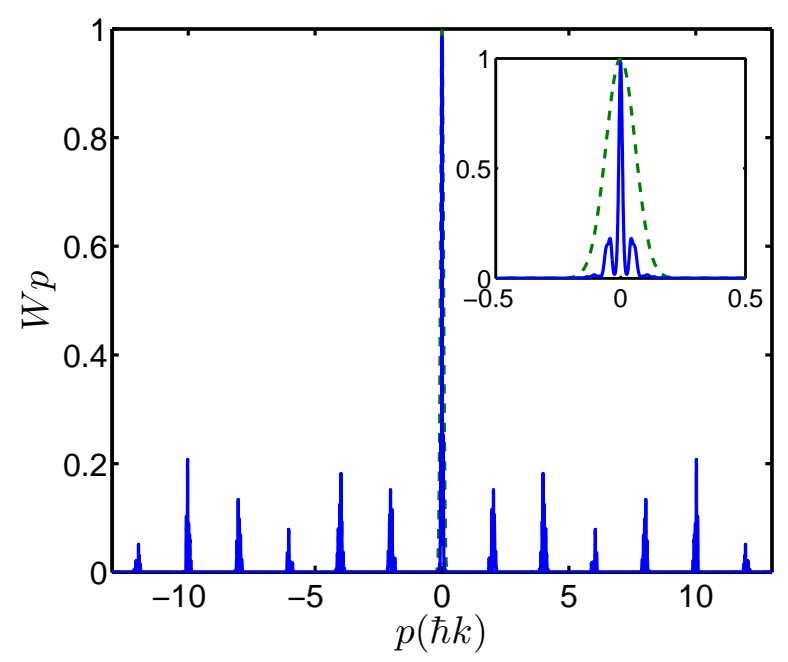

FIG. 1: (color online) The simulated momentum distribution after the kick sequence (full blue line) and the initial wave packet (dashed green line) are shown. The parameters are $N=10, \phi_{d} \sim 2.5$ and $\epsilon=2$. The inset shows a magnified view of the area around $p=0$.

tum space. The kicks are sufficiently short that the evolution due to the momentum can be ignored during the kicks, which is equivalent to the Raman-Nath regime. We start the simulation with a Gaussian wave packet with a width $(2 \sigma)$ of 0.1 recoils in momentum space.

The momentum distribution after the kick sequence can be written as $W_{p}(t)=|\langle p \mid \psi(t)\rangle|^{2} /|\langle 0 \mid \psi(0)\rangle|^{2}$. In Fig. 1 we show the resulting momentum distribution in the simulation after a pulse sequence as discussed. A much narrowed momentum distribution around $p=0$ after the kick sequence is observed, as the restitution of the wave function only works for very small values of $p$. The rest of the probability is transferred to higher momentum states, with offsets of $2 n \hbar k$ with integer $n$. Note however, that the probability for $p=0$ returns to its previous value. Martin et al. [17] have performed extensive simulations, showing that the $p=0$ peak gets narrower as the number of kicks $N$ in the sequence or the intensity increases. This does however mean that fewer atoms are in this peak than at lower intensities and/or lower kick number. Note that the final momentum distribution is not Gaussian. We therefore take the FWHM as the indicator for the width of the peak.

For the experiment, a reasonable number of atoms at small initial momenta is of paramount importance, and we therefore use a Bose-Einstein condensate (BEC) of $(F=1)^{87} \mathrm{Rb}$ at a temperature of $50 \mathrm{nK}$ as the source of atoms. The atoms are first captured and cooled in a magneto-optical trap (MOT) then transferred using a push laser to a second MOT in a second, connected chamber. An ultra-high vacuum is produced in the second chamber by an ion pump connected to it. The condensate is formed in a dipole trap overlapped with the second
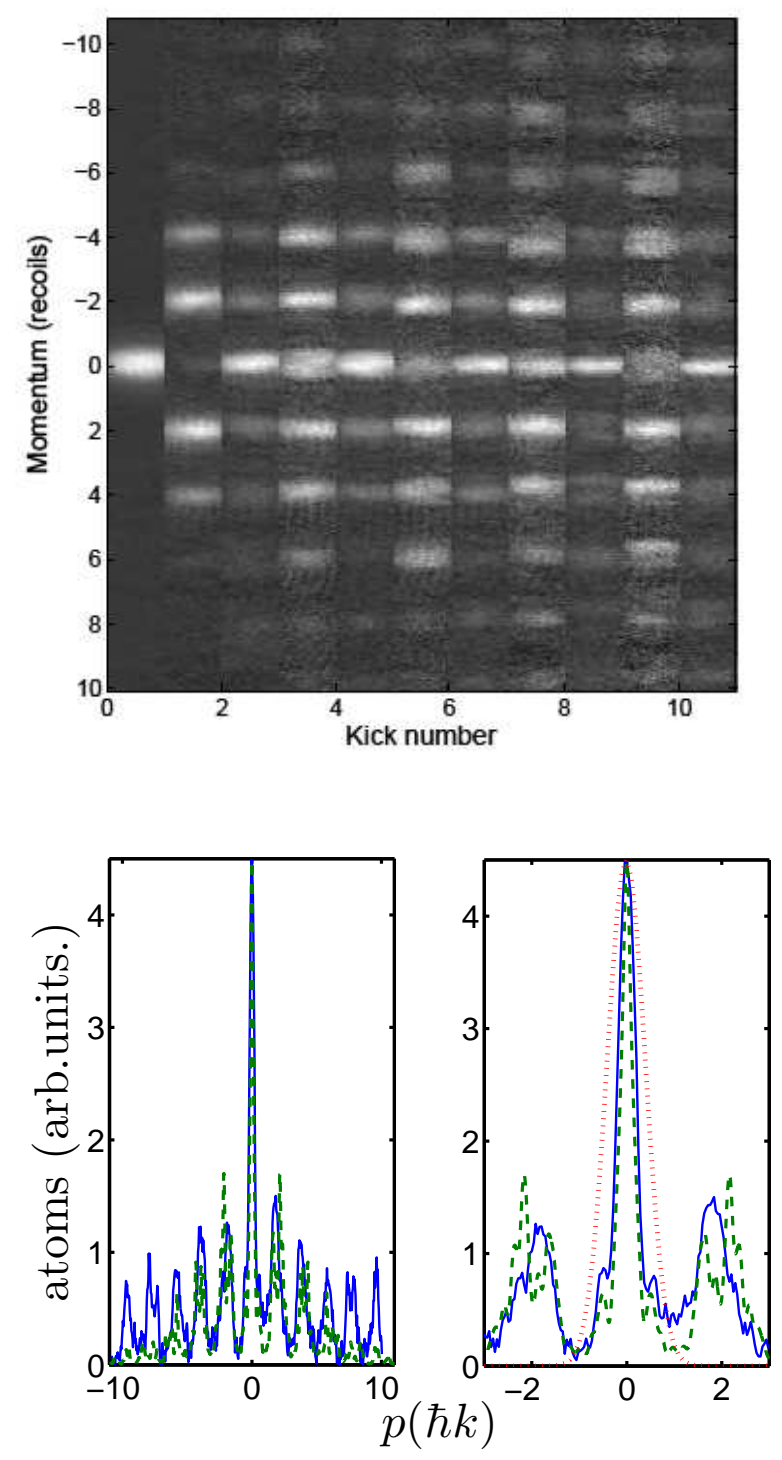

FIG. 2: (color online) (top) Absorption images of the momentum distribution before and after each kick (1-10). (bottom) The momentum distribution of the atoms at $N$ kicks (bottom left) for the experiment (solid blue) and the simulation (dashed green). The central part of the same curve is also shown (bottom right), with the initial state in (dotted red). The parameters are $N=10, \phi_{d} \approx 2$ and $\epsilon=1$.

MOT. The dipole trap is made by a pair of intersecting focused $\mathrm{CO}_{2}$ laser beams. A detailed description of the experimental setup can be found in 22].

We realize the AOKR by pulsing a near resonant optical standing wave, derived from a $780 \mathrm{~nm}$ diode laser onto a BEC of $\sim 10^{4}{ }^{87} \mathrm{Rb}$ atoms. The trap containing the BEC is turned off $500 \mu$ s before the kick sequence to reduce mean field effects. The kick laser is locked to the $S_{\frac{1}{2}}, F=2 \rightarrow P_{\frac{3}{2}}, F^{\prime}=3$ transition in ${ }^{85} \mathrm{Rb}$ isotope. Hence, the laser frequency is detuned by $2.45 \mathrm{GHz}$ from the relevant $F=1 \rightarrow F=2$ resonance 
frequency. The laser beam from the diode laser passes through a 50/50 beam splitter and the output beams are then passed through separate acousto-optic modulators (AOMs) for fast switching. After passing through the AOMs, the two beams pass through single mode optical fibres and are focussed onto the BEC from opposite directions to produce a standing wave. The beam diameter at the focus is $\sim 100 \mu \mathrm{m}$.

The laser pulses of the standing wave are the kicks that modify the momentum distribution of the atoms. The momentum distribution of the atoms after the kick sequence is measured by absorption imaging in time of flight, with a flight time of typically $8 \mathrm{~ms}$. Just prior to imaging, the atoms are optically pumped to the $F=2$ state by a $100 \mu$ s pulse on the $F=1 \rightarrow F^{\prime}=2$ repump transition. An absorption image is then obtained using a probe laser which is tuned to the $S_{\frac{1}{2}}, F=2 \rightarrow P_{\frac{3}{2}}, F^{\prime}=3$ transition. Typical results are shown in Fig. 2 (Top), where we show the absorption images of the momentum distribution before kicking, and the distribution after each kick. We sum the images along the rows to obtain a one-dimensional momentum distribution, which can be compared to the simulation. The resulting momentum distribution of the ensemble after 10 kicks with $\epsilon=1$ (bottom left) and the magnified view of central part of the same distribution are also shown in the figure. Note that some of the structure in the final distribution of Fig. 1 can be observed as a pedestal on the final zero momentum peak in the experiment.

After deconvolution with the original trap size, the width of the zero velocity peak after 10 kicks is $\sigma=0.21$ (recoils) as compared to $\sigma=0.43$ (recoils) for the initial momentum distribution without kicking. It should be noted that the height of the zero momentum peak after the full sequence does not return to the height of the original BEC peak, which is currently due to experimental limitations, mainly the resolution of the imaging system and the observable amount of absorption.

The Loschmidt time reversal works for a very narrow range of initial momenta, which depends on the value of $\epsilon$, the kick strength, and the total number of kicks. The more narrow this range, the fewer atoms are part of it. Hence, experimental limitations make it difficult to observe extremely narrow momentum distributions.

The BEC we produce in the experiment has a certain finite momentum width. In order to take into account this initial momentum width in the simulation, we divide the initial BEC distribution into a number of components and run the simulation for a range of initial momenta. We sum over the thus obtained momentum distributions, weighted by the initial BEC distribution. To account for the limited experimental resolution, we finally perform a convolution of the resultant momentum profile from the simulation with a Gaussian, with a width which is given by the experimental resolution. We can then determine the height of the momentum peaks obtained corresponding to various diffraction orders. In both the simulation and the experiment, the heights of the diffraction orders
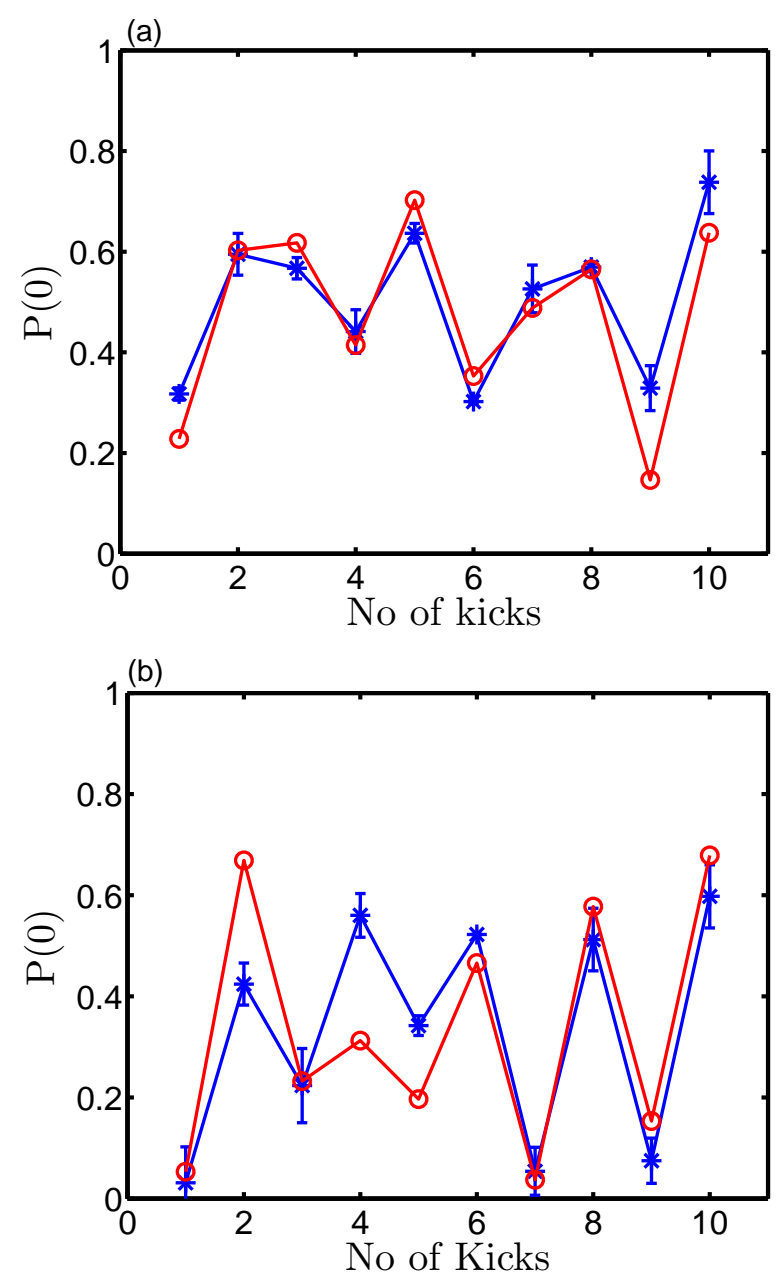

FIG. 3: (color online) The normalized height of the zeromomentum peak $\mathrm{P}(0)$, as obtained from the numerical simulation (red circles) and from the experiment (blue stars). Parameters are $\epsilon=1$ and $\phi_{d} \sim 2$ (a) and $\phi_{d} \sim 3$ (b).

is summed. We show the height of the zero momentum peak $\mathrm{P}(0)$, which corresponds to the number of atoms left with in the resolution of the experiment relative to this sum in Fig. 3, going through the kick sequence for two different kick strengths. The error bars in the experiment are determined by multiple runs of the experiment.

We observe good agreement between the simulation and the experiment in both cases. The normalized height is small after the first kick, as the probability is distributed across a number of momentum states. The height increases after the second kick, as the probability density for higher momentum states is less, which is clear from the absorption picture in Fig. 2. After the complete kick sequence, constructive interference of the wave function components leads to a strong peak at zero momentum. It should be noted that a maximum height does not necessarily correspond to the greatest number of "cold" atoms remaining, but rather to the most atoms that appear "cold" within the experimental resolution re- 


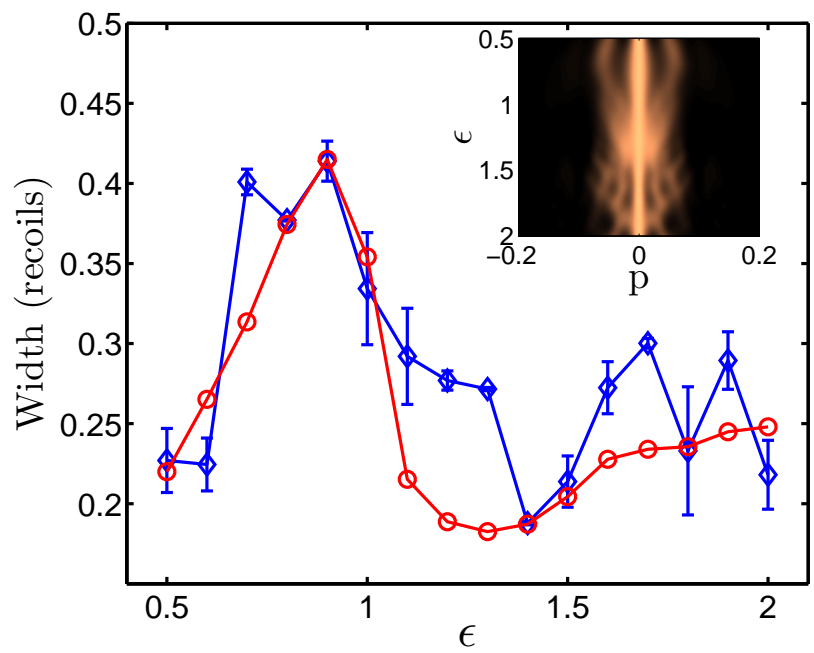

FIG. 4: (color online) Width of the zero momentum peak from experiment (blue diamonds) and simulation (red circles) for a range of $\epsilon$ values. Also shown (inset) is a theoretical simulation of the central part of the momentum distribution for the same range of $\epsilon$ values with no convolution applied. Other parameters are $\mathrm{N}=10$ and $\phi_{d} \sim 2$.

maining. From the comparison between the simulation and experimental results, we believe we have a good understanding of the parameters in the experiment, and we conclude that "Loschmidt" time reversal has been realized.

In Fig 4 we plot the width (FWHM) in recoils of the zero momentum peak as a function of $\epsilon$. The error bars shown are obtained by running the experiment a number of times. Initially we get some higher values of widths for the central peak, which starts to decrease around $\epsilon=1$ and gets narrower at $\epsilon \simeq=1.3$. It should be noted that there are fewer atoms where the peak is narrower. The results are more clear as seen in the theoretical simulation with no convolution applied in the inset of Fig, 4 , The return probability for the atoms is equal to 1 (in the simulation). As shown, the final width of the momentum distribution changes for different $\epsilon$. The sidelobes appearing for $\epsilon$ values between .5 and 1 , could be the evidence for the large widths obtained in this range. The lobes then starts to disappear and vanishes at $\epsilon \simeq$ 1.3 , which can be seen as a much narrower width of the momentum distribution for the corresponding value of $\epsilon$. The full structure appearing in the theory plot is difficult to resolve in the experiment because of limited experimental resolution, but the side-lobes can be observed in Fig. 2. The experimentally determined widths, however, follow the general trend of those from simulation after the convolution.

In conclusion, we have experimentally observed evidence for the time reversal of atomic matter waves in the ultra cold regime. As the time reversal only works for a narrow range of initial momenta, it shows a narrowing of the momentum distribution. We have shown this for a range of parameters in our experiment. In Future, it would be interesting to add a potential and see the effects of interactions between atoms as indicated by [23] on the phenomenon of time reversibility in quantum chaos.

Acknowledgements: The authors acknowledge the University of Auckland Research Fund and the Higher Eduction Commission for financial support. The authors would like to thank David Wardle for fruitful discussions.
[1] J. Loschmidt, Sitzungsberichte der Akademi der Wissenschaften II, Wein (1982).

[2] B. V. Chirikov, Phys. Rep 52, 263 (1979).

[3] I. P. Kornfeld, S. V. Fomin, and Y. G. Sinai, Ergodic theory II (Springer, N. Y., 1982).

[4] A. Lichetenberg and M. Lieberman, Regular and Chatoic dynamics (Springer, N. Y., 1992).

[5] M. Lieberman and A. Lichetenberg, Phys. Rev. A 5, 1852 (1972).

[6] B. V. Chirikov, F. M. Izrailev, , and D. L. Shepelyanski, Sov. Scient. Rev 2C, 209 (1981).

[7] M. G. Raizen, Adv. At. Mol. Opt. Phys. 41, 43 (1999).

[8] J. C. Robinson, C. Bharucha, F. L. Moore, R. Jahnke, G. A. Georgakis, Q. Niu, M. G. Raizen, and B. Sundaram, Phys. Rev. Lett 74, 3963 (1995).

[9] D. F. Shepelyansky, Physica D, 8 (2008).

[10] F. Haake, Quantum Signatures of Chaos (Springer, Berlin, 2001).

[11] H. Ammann, R. Gray, I. Shvarchuck, and N. Christensen, Phys. Rev. Lett 80, 4111 (1998).

[12] F. L. Moore, J. C. Robinson, C. F. Bharucha, B. Sundaram, and M. G. Raizen, Phys. Rev. Lett 75, 4598
(1995).

[13] F. Saif, Phys. Rep 419, 207 (2005).

[14] J. A. Currivan, A. Ullah, and M. D. Hoogerland, Euro. Phys. Lett 85, 30005 (2009).

[15] G. Casati and T. Prosen, Phys. Rev E59, R2516 (1999).

[16] G. Casati and T. Prosen, Physica D 131, 293 (1999).

[17] J. Martin, B. Georgeot, and D. L. Shepelyansky, Phys. Rev. Lett 100, 044106 (2008).

[18] J. Wang and J. Gong, Phys. Rev. A 77, 031405(R) (2008).

[19] S. Chu, J. E. Bjorkholm, A. Ashkin, J. P. Gordon, and L. W. Hollberg, Opt. Lett 11, 73 (1986).

[20] H. Ammann and N. Christensen, Phys. Rev. Lett 78, 2088 (1997).

[21] M. Weitz, T. Heupel, and T. W. Hansh, Appl. Phys. B 65, 713-724 (1997).

[22] Y. C. Wenas and M. D. Hoogerland, Review of Scientific Instruments 79, 053101 (2008).

[23] J. Martin, B. Georgeot, and D. L. Shepelyansky, Phys. Rev. Lett 101, 074102 (2008). 